\title{
Effects of Fibers on Color and Translucency Changes of Bulk-Fill and Anterior Composites after Accelerated Aging
}

\author{
Ali Riza Tuncdemir (iD) and Mehmet Esad Güven \\ Dentistry Faculty, Necmettin Erbakan University, Konya, Turkey \\ Correspondence should be addressed to Ali Riza Tuncdemir; alirizatuncdemir@gmail.com
}

Received 2 October 2017; Revised 3 December 2017; Accepted 13 December 2017; Published 28 January 2018

Academic Editor: Andrea Scribante

Copyright (C) 2018 Ali Riza Tuncdemir and Mehmet Esad Güven. This is an open access article distributed under the Creative Commons Attribution License, which permits unrestricted use, distribution, and reproduction in any medium, provided the original work is properly cited.

\begin{abstract}
The aim of this study was to determine the effects of glass and polyethylene fibers on the color and translucency change of bulkfill and anterior composites before and after artificial accelerated aging (AAA). Two types of teflon molds were used to fabricate samples which were $13 \mathrm{~mm}$ in diameter and, respectively, $2 \mathrm{~mm}$ and $4 \mathrm{~mm}$ in height. Polyethylene fiber (PF) and glass fiber (GF) were incorporated in the middle of the composite samples. Color and translucency changes of each composite were evaluated before and after AAA with spectrophotometer. ANOVA and Tukey's HSD post hoc statistical analysis were used at a significance level of 0.05. Before AAA (for anterior composites), there were no significant differences in $L^{*}$ and $b^{*}$ parameters among the three groups ( $p>0.05$ ); there were no significant differences in $L^{*}$ parameter between PF and GF groups or in TP between GF and control groups $(p>0.05)$ (for bulk-fill composites). After AAA, there were no significant differences in $L^{*}$ parameter between GF and control groups, in $a^{*}$ parameter between $\mathrm{PF}$ and control groups, in $b^{*}$ parameter among all groups, or in TP parameter between GF and control groups $(p>0.05)$. Fiber reinforcement led to color and TP change in both anterior and bulk-fill resin composites.
\end{abstract}

\section{Introduction}

Composite resin based materials have been widely used since their introduction to meet the growing demand for esthetic dental treatments [1]. The durability of composite resins is an important factor for their success. Applying fibers, for this reason, to reinforce the composite restorations started in the early 1990s [2]. Using a fiber reinforcement currently has a wide range of dental applications as in implant superstructure, removable partial denture, periodontal splints, and orthodontic retainers and it is an alternative to metal ceramic fix partial dentures [3]. Fibers used in this study were PF and GF. Ribbond-THM is a PF consisting of ultra-high strength braided polyethylene bondable fibers and is not impregnated with resin and must be saturated with an adhesive bonding agent before using. Interlig is a preimpregnated GF.

According to a study, 3 years' survival rates range up to $82.8 \%$ for metal ceramic, $88.5 \%$ for fiber reinforced, and $72,5 \%$ for ceramic resin-bonded prosthesis [4], and also other researches reported successful results and higher patient satisfaction with resin-bonded prosthesis for single tooth replacement than conventional fix partial denture [5].

The Fiber Reinforcement Composites (FRCs) consist of two parts. The fiber part reinforces the composites and provides stiffness; the matrix component (composite) supports the fibers, stabilizes their geometric orientation, and allows workability [3]. Different composite materials can be used for FRCs. It is important to determine which composites are more successful to ensure long-lasting FRC restorations. Color stability of composites affects its clinical longevity and if the color change results in patient dissatisfaction it can be concluded for total or partial replacement [6]. Several intrinsic and extrinsic reasons may cause composites to discolor. Extrinsic factors are related to plaque accumulation, absorption and accumulation of stains and the smoking habits; intrinsic factors are related to the chemical stability of the resin matrix and the matrix/particle interface [7]. Generally, manufacturers recommend that the composites should be placed in $2 \mathrm{~mm}$ increments to obtain sufficient light 
transmission and complete the curing of composite resin but using this incremental technique increases the possibility of air bubble inclusion or moisture contamination between increments of composites and also leads to waste of time [8].

Bulk-fill resin based composites (BRBCs) are innovative class of resin composites and produced to overcome such problems. These materials can be sufficiently light to cure up to $4 \mathrm{~mm}$ in a single increment with regard to manufacturers and cause low polymerization shrinkage [9]; the rate of filler content has been reduced to simplify deeper light transmission and particle sizes have been increased to improve the mechanical strength [10]. Recent studies have mostly focused on the depth of cure, degree of monomer conversion, and shrinkage stress, as well as microhardness and cytotoxicity of uncured monomers for BRBCs [11-13] and mechanical properties of FRCs [3]. The differences in filler content and composition are key to the optical feature of resin composites [14]. According to manufacturer both of these composites used in this study have patented innovative initiator system called "Radical Amplified Photopolymerization Technology" (RAP), to offer reduced curing time and excellent stability to ambient light while maintaining the superior esthetic and physical properties. In addition to this feature they include Supra-Nano Spherical filler (200 nm spherical SiO2-ZrO2) with quick curing time, 10 seconds, with a halogen light $\left(\geq 400 \mathrm{~mW} / \mathrm{cm}^{2}\right)$ and low polymerization shrinkage different from the other brands.

Translucency is a very important optical property to consider for the color of composite resins. It can be determined with the translucency parameter (TP) and can be described as a color difference in uniform thickness of a material over a white and black background [15]. The TP value is zero when the material is absolutely opaque. The greater the TP value is the higher the actual translucency of a material is. When a material's color has optimal translucency, the restoration will highly resemble the tooth structure and meet the esthetic requirements.

Color stability and translucency are very important for the esthetic restorations but there is no study about color stability of fiber-reinforced bulk filled composites. Therefore, the samples were subjected to artificial accelerated aging (AAA) in order to predict possible alterations on color and translucency change of the composites in a short time in this study.

Recent studies mostly focused on the depth of cure, degree of monomer conversion, and shrinkage stress as well as microhardness and cytotoxicity of uncured monomers for BRBCs $[16,17]$. The originality of this study was color stability and translucency is very important for the esthetic restorations but there is no study about effects of fibers on color and translucency changes of bulk-fill and anterior composites before and after AAA. Therefore, the purpose of this study was to determine the effects of fiber incorporation (glass and polyethylene fibers) and AAA on the color and translucency change of anterior composites and bulk-fill, respectively. The null hypothesis is that incorporation of fibers into the composites would not influence these composites' color and translucency.

\section{Materials and Methods}

In this in vitro experimental study, two types of fibers (glass and polyethylene) were incorporated into anterior and bulk-fill composites. Both composites' shades were A2. The characteristics and composition of the materials used in the study are shown in Table 1.

2.1. Sample Preparation. Two types of Teflon molds were used to fabricate samples which were $13 \mathrm{~mm}$ in diameter and, respectively, $2 \mathrm{~mm}$ and $4 \mathrm{~mm}$ in height. The spectrophotometer's reservoir diameter was $13 \mathrm{~mm}$, so this width was chosen to allow color measurement. The first layer of the anterior composites was prepared using the shallower $(2 \mathrm{~mm})$ mold to enable using incremental polymerization technique and then continued with deeper mold $(4 \mathrm{~mm})$ to complete samples. For bulk-fill composites only deeper $(4 \mathrm{~mm})$ mold was used to complete samples. Filled mold surface was covered with a Mylar film and the upper and bottom surfaces of the mold were covered by glass slabs before polymerization to produce a smooth surface and finger pressure was applied to extrude excess composite [18]. The samples were polymerized for 20 seconds using a light-emitting diode (LED) curing unit (Elipar S10; 3M ESPE; St. Paul, MN, USA) at a light intensity of $1200 \mathrm{~mW} / \mathrm{cm}^{2}$ and a wavelength of $430-480 \mathrm{~nm}$ (wavelength peak $455 \mathrm{~nm}$ ). The output of the curing light was tested with a radiometer $\left(1,200 \mathrm{~mW} / \mathrm{cm}^{2}\right)$. For fibers, both PFs (Ribbond-THM, Ribbond. Seattle, USA) (group PF) and resin impregnated GFs (glass fiber, Angelus, Sao Paulo, Brazil) (group GF) were cut with fiber scissors at $4 \mathrm{~mm}$ width, $10 \mathrm{~mm}$ length. Ten samples were prepared for each group and totally 60 samples were prepared for this test $(n=10)$.

2.1.1. For Control Groups. No fiber was added to the control groups. $2 \mathrm{~mm}$ Teflon mold was inserted into the $4 \mathrm{~mm}$ mold and the remaining $2 \mathrm{~mm}$ space was filled with composite and polymerized; then the remaining $2 \mathrm{~mm}$ composites were added for anterior composites. The residual $4 \mathrm{~mm}$ space of the mold was overfilled with bulk-fill composites as monoblock. The composites were cured for $20 \mathrm{~s}$ with using the same light curing unit.

2.1.2. For Polyethylene Fiber (PF) Groups. PF were impregnated with a bonding agent (Clearfil SE Bond) in a small plastic cup. To prepare PF-reinforced composite samples, another custom-made Teflon mold $(2 \mathrm{~mm}$ height, $13 \mathrm{~mm}$ diameter) was inserted into a $4 \mathrm{~mm}$ thick mold. After packing a $2 \mathrm{~mm}$ thick layer of composite, the custom-made $2 \mathrm{~mm}$ Teflon mold was removed. Without curing the bonding agent, PF was placed in the middle of the $2 \mathrm{~mm}$ height samples (Figures 1 and 2). After the mold was slightly overfilled with more composite resin, a Mylar strip was put on it and glass slab was clamped on upper surface to throw out excess resin. The composites were cured for $20 \mathrm{~s}$ using the same light curing unit.

2.1.3. For Glass Fiber (GF) Groups. Since the GFs were impregnated with resin, they were not subjected to an extra 


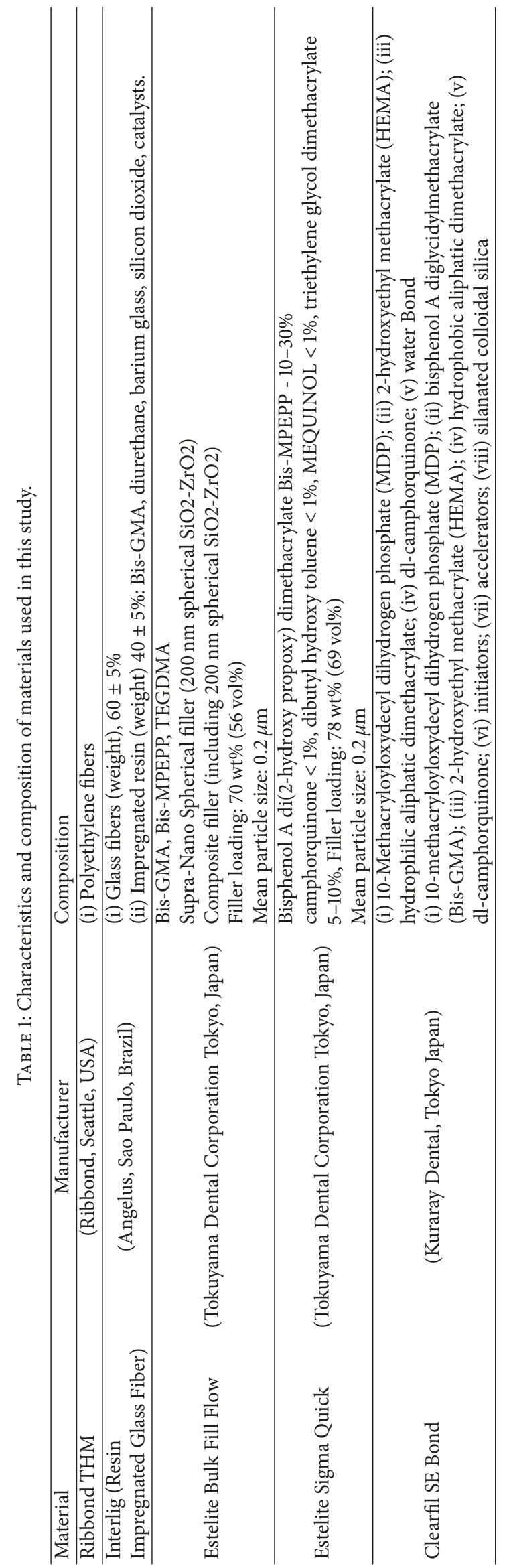



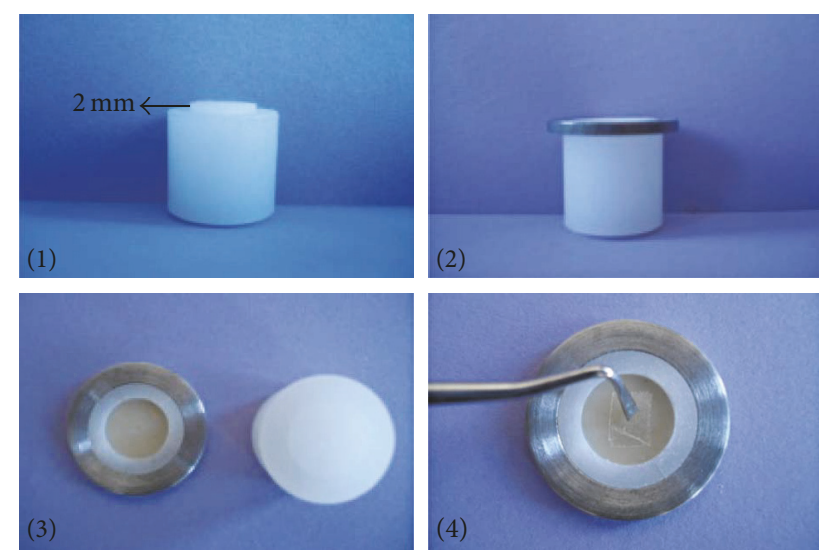

Figure 1: Preparation of samples.

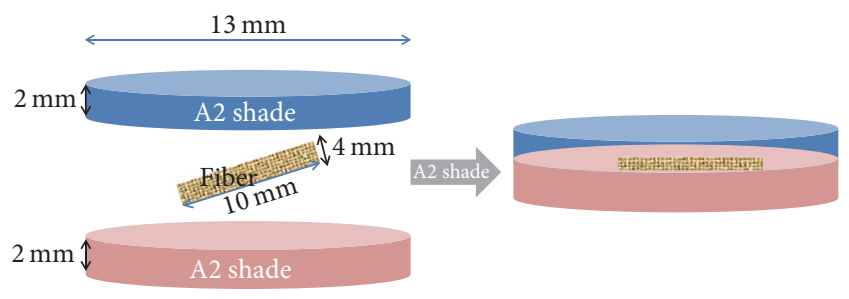

Figure 2: Final view of the samples in the mold.

bonding treatment. Composite resin filled and cured applications were performed as defined PF group.

2.2. Color and Translucency Measurement. After total 60 composite samples' polymerization, all samples were stored in distilled water at $37^{\circ} \mathrm{C}$ for 24 hours. Samples were stored in dark boxes until color was measured. Before placing the samples in the spectrophotometer (Lovibond ${ }^{\circledR}$ RT400 Tintometer Colour Measurement Amesbury, UK) it was calibrated according to per manufacturer's instructions and measured solely against white calibration tiles for color evaluation and against white and black calibration tiles for translucency measurements (mean calibration value study device's at D65 condition: white: $L^{*}=92,76, a^{*}=-1,16$, $b^{*}=0.70$; black: $\left.L^{*}=0.38, a^{*}=0.04, b^{*}=-0.18\right)$. The accurate positioning of samples was enabled by a way of custom jig. Three measurements were conducted at the center of each sample against a white and black background and the mean value was calculated. All samples were evaluated and measured by the same practitioner. Color alterations were determined using the Commission Internationale d'Eclairage $L^{*} a^{*} b^{*}$ color system (CIE $\left.L^{*} a^{*} b^{*}\right)$. Color changes were assessed using the following formula [19]:

$$
\begin{aligned}
& \Delta E=\left[\left(\Delta L^{*}\right)^{2}+\left(\Delta a^{*}\right)^{2}+\left(\Delta b^{*}\right)^{2}\right]^{1 / 2}, \\
& \Delta E=\left\{\left[L_{1}{ }^{*}-L_{2}{ }^{*}\right]^{2}+\left[a_{1}{ }^{*}-a_{2}{ }^{*}\right]^{2}+\left[b_{1}{ }^{*}-b_{2}{ }^{*}\right]^{2}\right\}^{1 / 2} .
\end{aligned}
$$

This formula was used twice in this study. First, it was used to determine the color differences between control group and fiber-reinforced composite groups at beginning
TABLE 2: National Bureau of Standards (NBS) units and the critical remarks of color change.

\begin{tabular}{lc}
\hline Colour difference & NBS unit \\
\hline Trace & $0-0.5$ \\
Slight & $0.5-1.5$ \\
Noticeable & $1.5-3.0$ \\
Appreciable & $3.0-6.0$ \\
Much & $6.0-12.0$ \\
Very much & $>12.0$ \\
\hline
\end{tabular}

Note. This table was extracted from our other study [15].

measurement. After that, it was used to determine the color changes of all experimental groups after AAA.

To determine the relationship between the amount of color alteration recorded on a spectrophotometer to the clinical environment, data was converted to the National Bureau of Standards (NBS) system units as follows:

$$
\text { NBS unit }=0.92 \times(\Delta E) .
$$

(See [20].) The defined critical remarks of color change according to the quantified NBS units are given in Table 2 [15]. The TP was calculated as the difference of color coordinate values obtained from the same specimen against black and white background as follows [21]:

$$
\begin{gathered}
\mathrm{TP}^{*}=\left[\left(L_{B}{ }^{*}-L_{W}{ }^{*}\right)^{2}+\left(a_{B}{ }^{*}-a_{W}{ }^{*}\right)^{2}\right. \\
\left.+\left(b_{B}{ }^{*}-b_{W}{ }^{*}\right)^{2}\right]^{1 / 2}
\end{gathered}
$$

where the subscript $B$ refers to color coordinate values obtained against the black background, and the subscript $W$ refers to the values obtained against the white background.

2.3. Artificial Accelerated Aging. After baseline color measurement, all specimens were aged for $150 \mathrm{~kJ} / \mathrm{m}^{2}$ according to accelerated aging conditions previously described [22]. With an accelerated aging chamber (Ci35 Weather-Ometer, Atlas Electric Devices, Chicago, IL, USA), other test parameters included sample surface temperature of maximum $65^{\circ} \mathrm{C}$ (light) and $38^{\circ} \mathrm{C}$ (dark) in relative environment humidity of $65 \%$. For rainy condition, surface temperature ranged from $18^{\circ} \mathrm{C}$ to $38^{\circ} \mathrm{C}$. Test cycle was $108 \mathrm{~min}$ light plus $65 \%$ humidity, 12 min light plus water spray, 108 min dark and 65\% humidity, and 12 min dark plus water spray for a total of 150 hours.

2.4. Statistical Analysis. Color difference and TP changes of anterior and bulk-fill composites were analyzed with oneway analysis of variance (ANOVA) and Tukey's HSD post hoc test according to fiber type at a significance level of 0.05 . To identify the existing differences, post hoc comparisons were performed using the Tukey HSD test and Tamhane's T2 tests. As one-way ANOVA assumes homogeneity of variance, Levene's test was used for homogeneity. The Tukey HSD post hoc test was used when equal variances and specimens' sizes were assumed and the Tamhane's T2 test was used for data where equal variances were not assumed. 
TABLE 3: Means and standard deviations of $L, a, b, \Delta L, \Delta a, \Delta b$, TP, and $\Delta E$ values and differences between groups for anterior composites.

\begin{tabular}{|c|c|c|c|c|c|c|c|c|c|c|}
\hline \multirow[b]{2}{*}{$L_{1}$} & \multicolumn{2}{|c|}{ Anterior PF } & \multicolumn{2}{|c|}{ Anterior GF } & \multicolumn{2}{|c|}{ Anterior control } & \multirow[t]{2}{*}{$p$} & \multirow[t]{2}{*}{$1-2$} & \multirow[t]{2}{*}{$2-3$} & \multirow[t]{2}{*}{$1-3$} \\
\hline & 64,25 & $(0,61)$ & 63,93 & $(0,67)$ & 64,31 & $(1,19)$ & & & & \\
\hline$L_{2}$ & 61,81 & $(0,67)$ & 62,02 & $(0,59)$ & 62,62 & $(0,98)$ & & & & \\
\hline$\Delta L$ & $-2,43$ & $(0,96)$ & $-1,90$ & $(0,87)$ & $-1,69$ & $(0,38)$ & & & & \\
\hline$a_{1}$ & 3,95 & $(0,29)$ & 4,24 & $(0,21)$ & 4,60 & $(0,15)$ & * & & $*$ & $*$ \\
\hline$a_{2}$ & 5,27 & $(0,36)$ & 5,31 & $(0,20)$ & 5,38 & $(0,19)$ & & & & \\
\hline$\Delta a$ & 1,32 & $(0,52)$ & 1,07 & $(0,16)$ & 0,78 & $(0,14)$ & * & & $*$ & $*$ \\
\hline$b_{1}$ & 17,76 & $(0,93)$ & 18,38 & $(0,73)$ & 18,42 & $(0,44)$ & & & & \\
\hline$b_{2}$ & 21,32 & $(1,67)$ & 21,30 & $(0,72)$ & 20,20 & $(0,62)$ & & & & \\
\hline$\Delta b$ & 3,56 & $(1,61)$ & 2,92 & $(0,82)$ & 1,78 & $(0,53)$ & * & & & $*$ \\
\hline $\mathrm{TP}_{1}$ & 6,53 & $(0,45)$ & 6,75 & $(0,32)$ & 7,42 & $(0,43)$ & * & & * & $*$ \\
\hline $\mathrm{TP}_{2}$ & 5,82 & $(0,50)$ & 5,71 & $(0,43)$ & 6,25 & $(0,50)$ & * & & * & \\
\hline$\Delta E$ & 4,57 & $(1,79)$ & 3,68 & $(1,07)$ & 2,61 & $(0,50)$ & $*$ & & & $*$ \\
\hline
\end{tabular}

${ }^{*} p<0.05 ; n=10$; anterior PF: incorporation of PF into the anterior composite (Group 1); anterior GF: incorporation of GF into the anterior composite (Group 2); anterior control: anterior composite control groups (Group 3); 1-2: statistical results between Group 1 and Group; 2-3: statistical results between Group 2 and Group 3; 1-3: statistical results between Group 1 and Group 3; $L_{1}, a_{1}, b_{1}$, and $\mathrm{TP}_{1}$ : values at baseline measurement; $L_{2}, a_{2}, b_{2}$, and $\mathrm{TP}_{2}$ : values after accelerated aging.

TABLE 4: Means and standard deviations of $L, a, b, \Delta L, \Delta a, \Delta b$, and $\Delta E$ values and differences between groups for anterior composites.

\begin{tabular}{|c|c|c|c|c|c|c|c|c|c|c|}
\hline & \multicolumn{2}{|c|}{ Bulk-fill PF } & \multicolumn{2}{|c|}{ Bulk-fill GF } & \multicolumn{2}{|c|}{ Bulk-fill control } & $p$ & $4-5$ & $5-6$ & $4-6$ \\
\hline$L_{1}$ & 67,12 & $(0,38)$ & 67,22 & $(0,42)$ & 68,28 & $(0,45)$ & $*$ & & * & $*$ \\
\hline$L_{2}$ & 65,22 & $(0,33)$ & 65,97 & $(0,55)$ & 65,92 & $(0,79)$ & * & $*$ & & * \\
\hline$\Delta L$ & $-1,91$ & $(0,19)$ & $-1,25$ & $(0,49)$ & $-2,36$ & $(0,75)$ & $*$ & $*$ & $*$ & \\
\hline$a_{1}$ & 3,63 & $(0,13)$ & 3,10 & $(0,25)$ & 3,66 & $(0,19)$ & $*$ & $*$ & $*$ & \\
\hline$a_{2}$ & 5,37 & $(0,20)$ & 5,06 & $(0,22)$ & 5,33 & $(0,29)$ & $*$ & $*$ & $*$ & \\
\hline$\Delta a$ & 1,74 & $(0,15)$ & 1,97 & $(0,27)$ & 1,67 & $(0,19)$ & $*$ & & * & \\
\hline$b_{1}$ & 19,34 & $(1,91)$ & 20,77 & $(1,34)$ & 21,76 & $(0,59)$ & $*$ & & & $*$ \\
\hline$b_{2}$ & 20,00 & $(0,82)$ & 20,08 & $(0,44)$ & 20,71 & $(0,70)$ & & & & \\
\hline$\Delta b$ & 0,66 & $(1,45)$ & $-0,70$ & $(1,25)$ & $-1,06$ & $(0,54)$ & $*$ & $*$ & & $*$ \\
\hline $\mathrm{TP}_{1}$ & 13,38 & $(0,95)$ & 14,51 & $(0,85)$ & 15,09 & $(0,53)$ & $*$ & $*$ & & $*$ \\
\hline $\mathrm{TP}_{2}$ & 11,57 & $(0,76)$ & 12,70 & $(0,53)$ & 13,02 & $(0,80)$ & $*$ & $*$ & & $*$ \\
\hline $\mathrm{DE}$ & 2,98 & $(0,44)$ & 2,71 & $(0,55)$ & 3,17 & $(0,48)$ & & & & \\
\hline
\end{tabular}

${ }^{*} p<0.05 ; n=10$; bulk-fill PF: incorporation of PF into the bulk-fill composite group (Group 4); bulk-fill GF: incorporation of GF into the bulk-fill composite group (Group 5); bulk-fill control: anterior composite control group (Group 6); 4-5: statistical results between Group 4 and Group 5; 5-6: statistical results between Group 5 and Group 6; 4-6: statistical results between Group 4 and Group 6; $L_{1}, a_{1}, b_{1}$, and $\mathrm{TP}_{1}$ : values at baseline measurement; $L_{2}, a_{2}, b_{2}$, and $\mathrm{TP}_{2}$ : values after accelerated aging.

\section{Results}

Table 3 shows the means $(\mathrm{M})$ and standard deviations (SD) of $L^{*}, a^{*}, b^{*}, \Delta L^{*}, \Delta a^{*}, \Delta b^{*}, \Delta E$, TP, and $p$ values of the anterior composites and also the differences between the experimental groups (EG). Before AAA, there were no significant differences in $L^{*}$ and $b^{*}$ parameters among the three EG $(p>0.05)$. However, the differences in TP and parameters between the groups were statistically significant $(p<0.05)$. After AAA, there were no significant differences in $L^{*}, a^{*}$, and $b^{*}$ parameters in all groups $(p>0.05)$. TP chance was statistically significant between GF and control groups. Color change was statistically significant between PF and control groups $(p<0.05)$.

Table 4 shows the $\mathrm{M}$ and $\mathrm{SD}$ of $L^{*}, a^{*}, b^{*}, \Delta L, \Delta a$, $\Delta b, \Delta E$, and TP values of the bulk-fill composites and the differences among the EG. Before AAA, there were no significant differences in $L^{*}$ parameter between PF and glass fiber groups, for a parameter between $\mathrm{PF}$ and control groups, for $b$ parameter between $\mathrm{PF}$ and GF and also GF and control groups, and for TP parameter, between GF and control groups $(p>0.05)$. There were statistically significant differences observed between other groups. After AAA, there were no significant differences in $L^{*}$ parameter between GF and control groups, for a parameter between $\mathrm{PF}$ and control groups, for $b^{*}$ parameter for all groups, and for TP parameter between GF and control groups $(p>0.05)$. There were statistically significant differences observed between other groups $(p<0.05)$.

Table 5 shows the color differences between the fiberreinforced groups and their respective control groups before AAA. As mentioned in this table, reinforcing with PF and GF to the anterior composites and reinforcing with GF fiber to the bulk-fill composites showed slight color change; on the 
TABLE 5: Color differences between control and fiber-reinforced groups in NBS units before aging.

\begin{tabular}{lcccccccc}
\hline Material & $\Delta L$ & $\Delta a$ & $\Delta b$ & TP & $\Delta E$ & NBS & Color difference \\
\hline Anterior PF & $-2,43$ & 1,32 & 3,56 & 6,52 & 0,93 & 0.86 & Slight \\
Anterior GF & $-1,9$ & 1,7 & 2,92 & 6,74 & 0,53 & 0,49 & Slight \\
Bulk-fill PF & $-1,91$ & 1,74 & 0,66 & 13,37 & 2,69 & 2,47 & Noticeable \\
Bulk-fill GF & $-1,25$ & 1,97 & $-0,7$ & 14,51 & 1,56 & 1,44 & Slight \\
\hline
\end{tabular}

other hand, reinforcing with PF to the bulk-fill composites showed noticeable color change.

\section{Discussion}

On the basis of the attained data, the null hypothesis tested in the present study was partly rejected. That incorporation of fibers would not change the color of composites' color which was rejected; however, the research hypothesis was accepted with respect to the fact that TP was changed after AAA.

The CIE Lab color system that defines color with using three parameters $\left(L^{*}, a^{*}\right.$, and $\left.b^{*}\right)$ was used in this study because of precise results for color parameters [23]. $L^{*}$ defines lightness/darkness ranging from white $(+)$ to black $(-), a^{*}$ defines red/green ranging from red $(+)$ to green $(-), b^{*}$ defines yellow $(+)$ and blue $(-)$, respectively, and $\Delta E$ shows color change of the material. It is a method for evaluating color differences based on human perception. Based on the human's eye ability, values $1<\Delta E<3.3$ are considered appreciable by skilled operators, but clinically acceptable, $\Delta E>3.3$ values are considered appreciable by nonskilled people and are, hence, not clinically acceptable [1]. In addition, in this study, NBS criteria were used to determine the relationship between the amount of color alteration recorded on a spectrophotometer and the clinical environment.

In this study, aging-dependent color differences are in accordance with some previous findings that accelerated aging resulting in the reduction in $L^{*}$ values and increase of $a^{*}$ and $b^{*}$ values $[24,25]$. It was surprising that in these studies aging to specimens was for 300 hours but in this study, aging time is 150 hours $\left(150 \mathrm{kj} / \mathrm{m}^{2}\right)$, but the results are the same. Therefore it is unnecessary to age 300 hours to find these results for these materials; on the other hand, different periods and aging methods should be conducted for other materials.

The color stability of composite resins can be related to the material properties, that is, composite matrix, filler composition (size and type volume of charged particles), matrixfiller interface, and degree of polymerization (proportion of remaining unreacted carbon-carbon bonds), shade and to the restorative techniques including the finishing and polishing procedures $[26,27]$. The polishing procedures were not investigated in this study; so to achieve the smoothest surface and to standardize the specimens, a Mylar strip was used during light-polymerizing and also to represent clinical situations when matrices were used [28].

Before AAA, incorporation of fibers in anterior composites changed their colors $\Delta E=0,53$ (anterior GF) and $\Delta E$ $=0,93$ (anterior PF) and also with these color differences, they were considered clinically slight $(\Delta E<1.5)$; for bulk-fill composites $\Delta E=1,56$ (bulk-fill GF) and $\Delta E=$ 2,69 (bulk-fill PF) these color differences were noticeable $(\Delta E>1.5)$. As mentioned in Results, incorporation of fibers affected anterior composites clinically slightly and bulk-fill composites clinically noticeably. This discrepancy can be more translucency of bulk-fill composites.

According to a study [29], bulk-fill composites had similar color stability to hybrid composite after 40 days of AAA, which is similar to Tiba and others [30], but in this study, after accelerated aging, both of the composites showed clinically noticeable color change and also bulk-fill composites became more colorful (2,92 NBS units) than anterior composites (2,40 NBS units) without fibers. This can arise from more than one factor. Both of the composites contain silica-zirconia and composite filler but in different ratios, as showed in Table 1. Filler weight and percentage of the bulk-fill composites are lower than anterior composites. It may result in surface degradation of the material and absorbing more water, and then greater water sorption provides the composite with lower color stability, due to the increase in free volume of the formed polymer and, as a result, greater space for the water molecules emerges to diffuse into the polymeric network, contributing to degradation of the material [31]. The literature is confusing about the effects of the filler size of the composites on color [22, 31-33]. On the other hand, according to a study, monomer content and surface roughness affect discoloration of composites more than size of the filler particles does [34]. Hydrophilicity of the bulk-fill composite monomers may be more than anterior composites; for example, triethylene glycol dimethacrylate (TEGDMA) absorbs water more than a bisphenol glycidyl methacrylate does (Bis-GMA) and these proportions are also important [35]. In absence of pigments, degree of polymerization (proportion of remaining unreacted carbon-carbon bonds) and greater translucency in bulk-fill composites may be one of the other discoloration factors so discoloration is a multifactorial problem.

After AAA, the FRC and non-FRC groups of both anterior control composites and all of the bulk-fill composites showed clinically noticeable color changes in the range of 1.5-3 NBS units and also anterior PF and GF groups showed clinically appreciable color changes in the range of 3-6 NBC units in this study.

According to this study, the most color changes were observed in anterior PF composites (4.2 NBC units); the least were observed in anterior control group (2,40 NBC units). Thicknesses were chosen nearly the same in specimens (Ribbond-THM $=0,18 \mathrm{~mm}$, glass fiber $=0,2 \mathrm{~mm}$ ) in order not to affect results. Therefore, differences in their chemical 
structures and preparation procedures could be the reason why PF-reinforced composites exhibited greater color change than GF-reinforced composites did. PF is hand fabricated and GF is preimpregnated fibers by manufacturers. Improperly saturated fibers $(\mathrm{PF})$ may cause voids in FRC and enhance water sorption, and consequently it became more colorful $[18,36]$. On the other hand, this finding could be the result of superior adaptation with minimal space between the composite and the GFs. The refractive index of glass-fibers is different from that of the surrounding composite matrix along with its fillers and opacifiers and favors light penetration through composite, so it can be observed as light-colored compared to control groups. A previous study confirms this result [37].

According to our study, bulk-fill composites' TP were higher than anterior composites and adding to fibers decreased the TP values of specimens. Between the fiber groups, PF groups have less TP values than GF groups. After AAA, TP values of the all groups decreased. According to studies $[22,38]$ high temperature during accelerated aging could have increased the degree of conversion, leading to a change in the refractive index of the matrix. This, in turn, would make the material less translucent as our study increased scattering as a result. On the other hand, in Korkmaz Ceyhan et al. [39] study, obviously in contrast to our study, AAA did not influence the translucency of composites; it may arise from discrepancies of the shade and composites' content.

There were some limitations in this study. This was an in vitro study and influence of brushing and acids from foods and beverages effects on color stability of samples were not tested. These factors can cause major color change in composites in clinical practice. Examining all these parameters in future studies can lead to providing more precise results as well as in vivo studies.

\section{Conclusions}

According to present findings, the following conclusions were drawn.

(1) Fiber reinforcement led to color and TP change in both anterior and bulk-fill resin composites, but the color changes were below the visual perceptibility threshold $(\Delta E>3.3)$.

(2) PFs resulted in more colors and TP change than GFs after incorporation into the composite resins. Therefore, laboratory processed fibers would achieve better optimization of esthetics due to better processing and less voids, they can be preferred in esthetic area. PF can be used in nonesthetic area (palatal and posterior regions).

(3) After AAA, FRC and non-FRC groups of both composite materials became darker ( $-L$ values), more reddish $(+a)$, and more yellowish $(+b)$.

(4) After AAA, anterior control and all of the bulk-fill composite groups showed clinically noticeable color changes in the range of 1.5-3 NBS units and also anterior PF and GF groups showed clinically appreciable color changes in the range of 3-6 NBC units.

(5) After AAA, the most color change was observed at anterior PF group (appreciable, 4,57 NBS units); the least was observed in anterior control groups (noticeable, 2,61 NBS units).

(6) After AAA, TP decreased in all groups; before and after AAA, bulk-fill composites were more translucent than anterior composites, and GF fibers were more translucent than PF fibers.

\section{Conflicts of Interest}

The authors declare that they have no conflicts of interest.

\section{References}

[1] A. Catelan, A. L. F. Briso, R. H. Sundfeld, M. C. Goiato, and P. H. Dos Santos, "Color stability of sealed composite resin restorative materials after ultraviolet artificial aging and immersion in staining solutions," Journal of Prosthetic Dentistry, vol. 105, no. 4, pp. 236-241, 2011.

[2] D. Wolff, T. Wohlrab, D. Saure, J. Krisam, and C. Frese, "Fiberreinforced composite fixed dental prostheses: A 4-year prospective clinical trial evaluating survival, quality, and effects on surrounding periodontal tissues," Journal of Prosthetic Dentistry, 2017.

[3] N. Tanoue, T. Sawase, H. Matsumura, and J. F. McCabe, "Properties of indirect composites reinforced with monomer-impregnated glass fiber," Odontology, vol. 100, no. 2, pp. 192-198, 2012.

[4] M. Miettinen and B. J. Millar, "A review of the success and failure characteristics of resin-bonded bridges," British Dental Journal, vol. 215, no. 2, p. E3, 2013.

[5] D. S. Thoma, I. Sailer, A. Ioannidis, M. Zwahlen, N. Makarov, and B. E. Pjetursson, "A systematic review of the survival and complication rates of resin-bonded fixed dental prostheses after a mean observation period of at least 5 years," Clinical Oral Implants Research, 2017.

[6] H. E. Strassler and C. L. Serio, "Single-visit natural tooth pontic fixed partial denture with fiber reinforcement ribbon," Compendium of Continuing Education in Dentistry, vol. 25, no. 3, pp. 224-230, 2004.

[7] A. B. Souza et al., "Color stability of repaired composite submitted to accelerated artificial aging," General Dentistry, vol. 60, no. 5, pp. e321-e325, 2012.

[8] Z. Tarle, T. Attin, D. Marovic, L. Andermatt, M. Ristic, and T. T. Tauböck, "Influence of irradiation time on subsurface degree of conversion and microhardness of high-viscosity bulk-fill resin composites," Clinical Oral Investigations, vol. 19, no. 4, pp. 831840, 2015.

[9] N. Ilie, S. Bucuta, and M. Draenert, "Bulk-fill resin-based composites: an in vitro assessment of their mechanical performance," Operative Dentistry, vol. 38, no. 6, pp. 618-625, 2013.

[10] U. Koc-Vural, I. Baltacioglu, and P. Altinci, "Color stability of bulk-fill and incremental-fill resin-based composites polished with aluminum-oxide impregnated disks," Restorative Dentistry \& Endodontics, vol. 42, no. 2, pp. 118-124, 2017.

[11] R. Z. Alshali, N. A. Salim, J. D. Satterthwaite, and N. Silikas, "Long-term sorption and solubility of bulk-fill and conventional resin-composites in water and artificial saliva," Journal of Dentistry, vol. 43, no. 12, pp. 1511-1518, 2015.

[12] B. M. Fronza, F. A. Rueggeberg, R. R. Braga et al., "Monomer conversion, microhardness, internal marginal adaptation, and shrinkage stress of bulk-fill resin composites," Dental Materials, vol. 31, no. 12, pp. 1542-1551, 2015. 
[13] C. M. P. Rosatto, A. A. Bicalho, C. VerÃssimo et al., "Mechanical properties, shrinkage stress, cuspal strain and fracture resistance of molars restored with bulk-fill composites and incremental filling technique," Journal of Dentistry, vol. 43, no. 12, pp. 1519-1528, 2015.

[14] M. Arregui, L. Giner, M. Ferrari, M. Vallés, and M. Mercadé, "Six-month color change and water sorption of 9 new-generation flowable composites in 6 staining solutions," Brazilian Oral Research, vol. 30, no. 1, p. el23, 2016.

[15] A. R. Tuncdemir and F. Aykent, "Effects of fibers on the color change and stability of resin composites after accelerated aging," Dental Materials, vol. 31, no. 5, pp. 872-878, 2012.

[16] T. T. Tauböck, Z. Tarle, D. Marovic, and T. Attin, "Pre-heating of high-viscosity bulk-fill resin composites: Effects on shrinkage force and monomer conversion," Journal of Dentistry, vol. 43, no. 11, pp. 1358-1364, 2015.

[17] N. Ilie and K. Stark, "Effect of different curing protocols on the mechanical properties of low-viscosity bulk-fill composites," Clinical Oral Investigations, vol. 19, no. 2, pp. 271-279, 2015.

[18] S. Sampath and G. S. Ramachandra, "Effects of glass fibers on light transmittance and color of fiber-reinforced composite," Dental Materials, vol. 24, no. 1, pp. 34-38, 2008.

[19] H. C. Kucukesmen, A. Usumez, N. Ozturk, and E. Eroglu, "Change of shade by light polymerization in a resin cement polymerized beneath a ceramic restoration," Journal of Dentistry, vol. 36, no. 3, pp. 219-223, 2008.

[20] M. Nagakura, Y. Tanimoto, and N. Nishiyama, "Color stability of glass-fiber-reinforced polypropylene for non-metal clasp dentures," Journal of Prosthodontic Research, 2017.

[21] H.-K. Hyun, C. K. Christoferson, C. S. Pfeifer, C. Felix, and J. L. Ferracane, "Effect of shade, opacity and layer thickness on light transmission through a nano-hybrid dental composite during curing," Journal of Esthetic and Restorative Dentistry, vol. 29, no. 5, pp. 362-367, 2017.

[22] K. A. Schulze, S. J. Marshall, S. A. Gansky, and G. W. Marshall, "Color stability and hardness in dental composites after accelerated aging," Dental Materials, vol. 19, no. 7, pp. 612-619, 2003.

[23] W. M. Johnston, "Color measurement in dentistry," Journal of Dentistry, vol. 37, no. 1, pp. e2-e6, 2009.

[24] G. Saygili, S. Şahmali, and F. Demirel, "Colour stability of porcelain repair materials with accelerated ageing," Journal of Oral Rehabilitation, vol. 33, no. 5, pp. 387-392, 2006.

[25] I. Karaokutan, T. Y. Savas, F. Aykent, and E. Ozdere, "Color stability of CAD/CAM fabricated inlays after accelerated artificial aging," Journal of Prosthodontics, vol. 25, no. 6, pp. 472-477, 2015.

[26] H. M. Barakah and N. M. Taher, "Effect of polishing systems on stain susceptibility and surface roughness of nanocomposite resin material," Journal of Prosthetic Dentistry, vol. 112, no. 3, pp. 625-631, 2014.

[27] F. D. C. P. Pires-De-Souza, L. D. F. R. Garcia, H. M. Hamida, and L. A. Casemiro, "Color stability of composites subjected to accelerated aging after curing using either a halogen or a light emitting diode source," Brazilian Dental Journal, vol. 18, no. 2, pp. 119-123, 2007.

[28] M. A. Arocha, J. R. Mayoral, D. Lefever, M. Mercade, J. Basilio, and M. Roig, "Color stability of siloranes versus methacrylatebased composites after immersion in staining solutions," Clinical Oral Investigations, vol. 17, no. 6, pp. 1481-1487, 2013.

[29] M. E. Gezawi, D. Kaisarly, H. Al-Saleh, A. ArRejaie, F. AlHarbi, and K. H. Kunzelmann, "Degradation potential of bulk versus incrementally applied and indirect composites: Color, microhardness, and surface deterioration," Operative Dentistry, vol. 41, no. 6, pp. e195-e208, 2016.

[30] A. Tiba, G. G. Zeller, C. G. Estrich, and A. Hong, "A laboratory evaluation of bulk-fill versus traditional multi-increment-fill resin-based composites," Journal of the American Dental Association (1939), vol. 144, no. 10, pp. 1182-1183, 2013.

[31] J. L. Ferracane, "Hygroscopic and hydrolytic effects in dental polymer networks," Dental Materials, vol. 22, no. 3, pp. 211-222, 2006.

[32] D. C. Tornavoi, J. A. M. Agnelli, H. Panzeri, and A. C. D. Reis, "Color change of composite resins subjected to accelerated artificial aging," Indian Journal of Dental Research, vol. 24, no. 5, pp. 605-609, 2013.

[33] Y.-R. Suh, J.-S. Ahn, S.-W. Ju, and K.-M. Kim, "Influences of filler content and size on the color adjustment potential of nonlayered resin composites," Dental Materials, vol. 36, no. 1, Article ID dmj/2016-083, pp. 35-40, 2017.

[34] N. Gönülol and F. Yilmaz, "The effects of finishing and polishing techniques on surface roughness and color stability of nanocomposites," Journal of Dentistry, vol. 40, supplement 2, pp. e64-e70, 2012.

[35] R. Lagocka, K. Jakubowska, D. Chlubek, and J. BuczkowskaRadlinska, "The influence of irradiation time and layer thickness on elution of triethylene glycol dimethacrylate from SDR bulk-fill composite," BioMed Research International, vol. 2016, Article ID 3481723, 10 pages, 2016.

[36] R. Mosharraf and S. Torkan, "Fracture resistance of composite fixed partial dentures reinforced with Pre-impregnated and Non-impregnated fibers," Journal of Dental Research, Dental Clinics, Dental Prospects, vol. 6, no. 1, pp. 12-16, 2012.

[37] M. H. Tabatabaei, "Effect of Accelerated Aging on Color Change of Direct and Indirect Fiber-Reinforced Composite Restorations," J Dent (Tehran, vol. 13, no. 3, pp. 168-175, 2016.

[38] Y.-K. Lee and J. M. Powers, "Color changes of resin composites in the reflectance and transmittance modes," Dental Materials, vol. 23, no. 3, pp. 259-264, 2007.

[39] Y. Korkmaz Ceyhan, J. C. Ontiveros, J. M. Powers, and R. D. Paravina, "Accelerated aging effects on color and translucency of flowable composites," Journal of Esthetic and Restorative Dentistry, vol. 26, no. 4, pp. 272-278, 2014. 


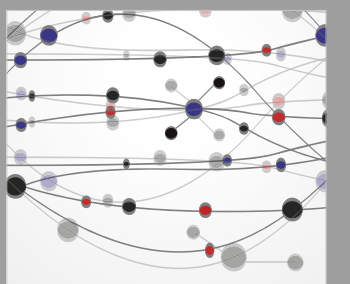

The Scientific World Journal
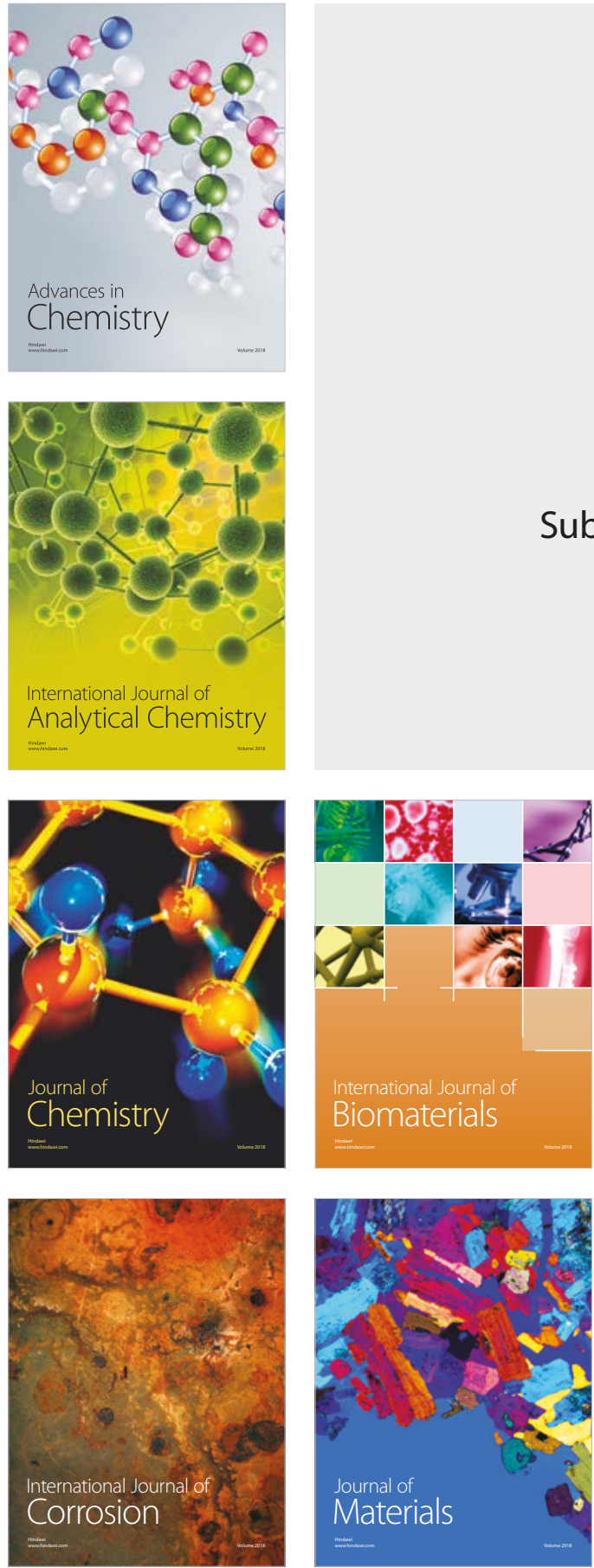

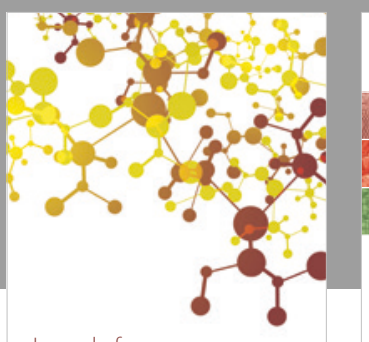

Journal of

Applied Chemistry
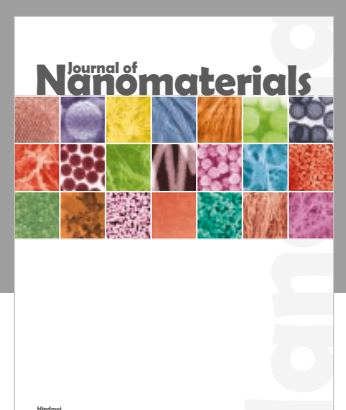

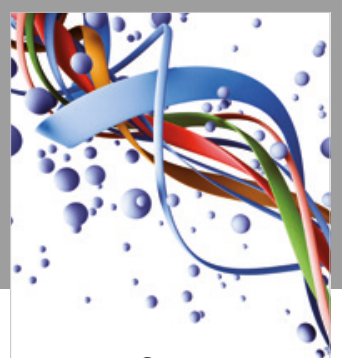

Scientifica

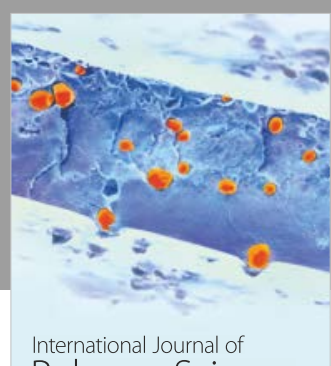

Polymer Science

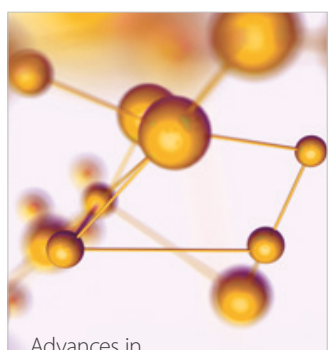

Physical Chemistry
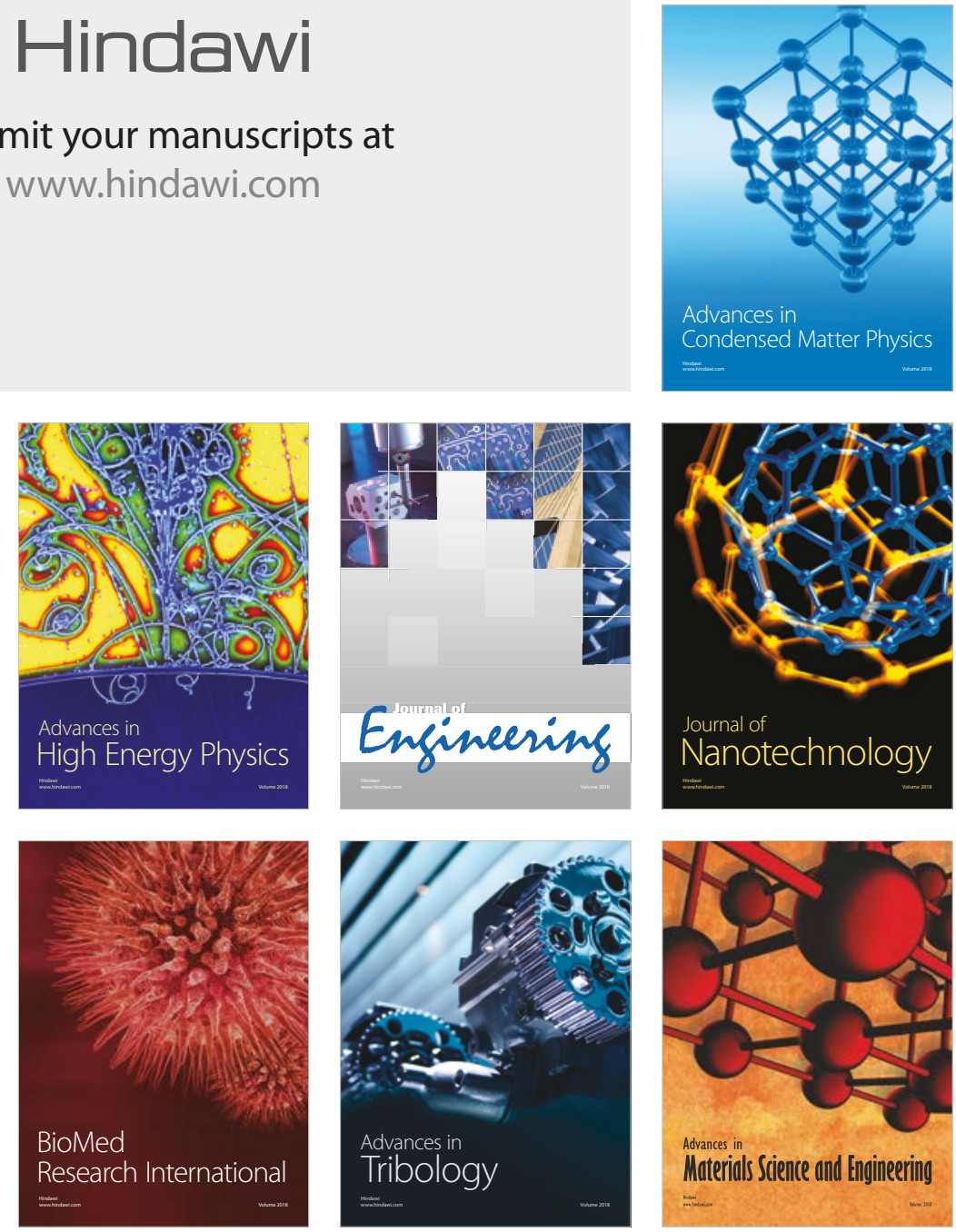